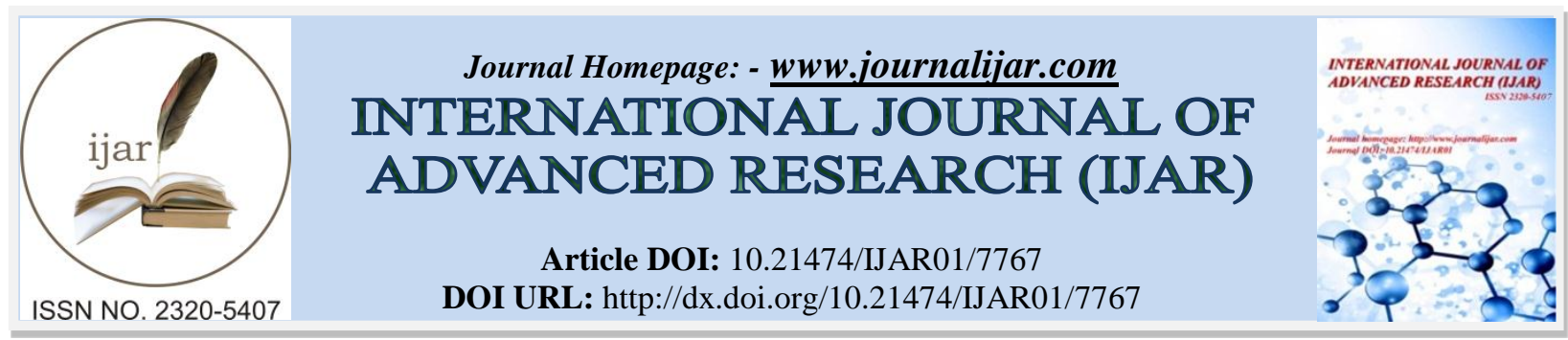

RESEARCH ARTICLE

\title{
A PERSPECTIVE: AN EXPLORATION OF THE MENTAL HEALTH EFFECTS OF MALNUTRITION FROM GESTATION TO TWO YEARS OF AGE.
}

\author{
Brandi Phillips.
}

MA Saybrook University.

\section{Manuscript Info}

Manuscript History

Received: 25 July 2018

Final Accepted: 31 August 2018

Published: September 2018

Keywords:-

Iron Deficiency, Protein-Energy

Deficiency, Fatty Acids, Dha, Omega-3.

\section{Abstract}

Nutrients, like that of iron, protein, and fatty acids DHA and omega-3, are essential in brain development from the beginning of gestation through 2 years of age. Deficiencies in these nutrients during the critical brain development stage can cause lasting mental health effects like slow gross motor function, depression, attention deficits, and impulse control leading to conduct problems. Although supplementation of these essential nutrients are suggested, studies show the damage done may not be reversible. Alternative psychosocial interventions are suggested.

Copy Right, IJAR, 2018,. All rights reserved.

\section{Introduction:-}

A Review: An Exploration of the Mental Health Effects of Malnutrition from Gestation to Two Years of Age

More than three million deaths each year of children five years or younger can be attributed to deficiencies of essential vitamins and minerals (Black et al., 2013). Black et al. (2013) claimed the crucial window of opportunity to prevent these deaths is the first 1,000 days or pregnancy through the first two years of life. Malnourishment during this crucial window can result in great cognitive deficits.

In an epidemiologic analysis by Martorell et al. (2010), it was found that weight attained during the first two years of life helped to predict educational outcomes. Conversely, weight attained between ages two and four does not affect educational outcomes. Also, moderate to severe malnutrition in the first year of life has been associated with subsequent attention problems, lower social status, and standard of living, even 37-43 years later (Prado and Dewey, 2014). The purpose of this paper is to explore the relationship between nutritional deficits from gestation through the first two years of life and subsequent mental health issues.

\section{Brain Development during Gestation and Infancy}

According to Prado and Dewey (2014) nutrient deficiencies are more important during gestation and infancy than any other time. They explained that about 22 days after conception the neural tube is developed, which later transforms into the spinal cord and brain. Seven weeks after conception, cell division begins in the neural tube. Once a neuron is created, it moves to its home in the brain and begins to grow axons and dendrites. These branches connect with other cells. These connections are synapses, and nerve signals travel through synapses. This growth for brain communications starts during gestation and continues at a rapid pace throughout infancy. 
The growth of the brains of neonatal infants through two years old was examined in a longitudinal quantitative study by Knickmeyer et al. (2008). They found the rate at which the brains grows in the first year of life makes this time the most critical period for disruption in the developmental process. In the study, magnetic resonance imaging (MRI) was done on 84 neonates, ages 2-4 weeks, 35 one-year-olds, and 26 two-year-olds. The images concluded that the brain grows $101 \%$ in the first year of life and an additional $15 \%$ in the second year. Grey matter, which will be discussed more in this paper, grew 149\% in the first year. Of that, the cerebellum increased $240 \%$, which is responsible for coordinating and regulating muscular activities. The lateral ventricles in the hemispheres increased by $280 \%$ in the first year then decreases in the second year of life. Lateral ventricles, if not regulated properly through nutrition, became enlarged. Enlarged ventricles are found in schizophrenia and bipolar disorders. Lastly, the hippocampus was found to grow an average of $13 \%$ from the first year to the second year of life. The hippocampus is charge of emotion, learning and memory, and regulation of the autonomic nervous system (Knickmeyer et al., 2008, p. 12187-12180). The growth of the brain in the first two years effects the ability of the brain to organize the tremendous input of information that comes with the first experiences.

Nutrient deficiencies effect the way the brain organizes responses to experiences. During the first two years of life, an infant may not have experienced a sensation or situation before. This information processed from these new experiences are process with the particular response experienced at the time. Prado and Dewey (2014) characterized these responses as experience-expectant and experience-dependent processes. Experience-expectant are the responses from the brain based on universal environmental situations and stimuli. Experience-dependent processes are unique to the individual. If the dendrites are not branched properly and the synapsis are not dense enough because of nutrient deficiencies while forming, the brain cannot process experience-dependent information as well as it would have with proper formation. Thus, the individual may not be able to adapt to their environment and thrive. So, Essential macronutrients, like proteins and fatty acids, and micronutrients, like iron, are needed for brain maturation and long-term life success.

\section{Iron Deficiency during Gestation and Infancy}

According to Black et al. (2011), there is evidence to relate iron deficiencies in infants with slow brain maturation. Low levels of cerebral iron decreases myelination and dopaminergic functioning. Myelination is the formation of the fatty protective layer of the myelin. Dopamine in s neurotransmitter, or a chemical signal sent out by neurons in the brain. Dopaminergic functioning is how well the dopamine is sent throughout the proper channels to the receptor sites (Lackner et al., 2010). This processing, or metabolism, is essential to cognitive process. Dopamine metabolism plays an important role in memory, motivation, attention, and perception (Black, et. al., 2011).

Black et al. (2011) claimed iron deficiency is the most common deficiency of a single nutrient in the world. Iron deficiency affects $50 \%$ of women of childbearing age and is even higher, up to $80 \%$, in low to middle income countries. Most infants are protected from iron deficiency through the first four to six months of life, but their vulnerability to increases as they approach a year because their need for iron increases beyond what breastmilk is able to provide. The prevalence of this micronutrient deficiency in mothers directly effects the brain development of babies in utero and creates an existing deficiency into infancy if not provided through feedings.

Children under two years are exceptionally vulnerable to experiencing the effects of iron deficiency because of the rapid brain developments during this time. These developments set up the pathways for proper formation of the frontal lobe of the brain and dopamine functioning (Lackner et al., 2010). Black et al. (2011) went on to explain that iron deficiency may inhibit myelination and change dopamine and norepinephrine metabolism. Prado and Dewey (2014) clarified that iron deficiency decreases the amount of dopamine D2 receptors and lowers the density of the dopamine transporters. If the density of the transporters is lowered, the neurotransmitter activity decreases. Iron deficiency and depression have been linked because they share dopamine neurotransmitter pathways (Black et al., 2011).

Iron deficiency and iron deficiency anemia generally are concurrent with poverty and a lower access to iron-rich foods. Without iron-rich foods or formula, iron deficiencies during gestation and infancy has been linked to "truncated dendritic branching in the hippocampus" which leads to a decreased size of the hippocampus. The hippocampus is the part of the brain responsible for learning and memory (Prado \& Dewey, 2014, p. 269). According to Black et al. (2011), infants evaluated and found anemic at nine months of age, through the Florida Special Nutritional Supplemental Program for Women, Infants, and Children (WIC), were also found to have academic problems at the age of 10 compared to those who were not anemic. Both Black et al. (2011) and Prado 
and Dewey (2014) cited a Lozoff et al. (2006) longitudinal study in which Costa Rican adolescents with severe iron deficiencies during infancy "showed poorer executive functioning and poorer recognition memory...and more internalizing and externalizing behavior problems compared with children who were iron sufficient as infants" (Black et al., 2011, p. 565).

The lack of iron-rich foods available throughout the world enables anemia, severe iron deficiency, to be one the leading causes of malnutrition in the world and attributing to $45 \%$ of the deaths of children under 5 years old (Black et al., 2013). With damage done to the brain in the crucial developmental period though, cognitive development is already slowed. Unfortunately, another compounding issue with anemia is protein deficiency since many of the iron-rich food sources are also high in protein. Protein is essential for creating brain cells during the first 1000 days of life.

\section{Protein Deficiency during Infancy}

Kulkarni and Metgund (2014) cited protein-energy deficiency to correlate with an insufficient intake of protein-rich foods. This usually happened in infancy because of lack of access to protein-rich foods after six months or lack of education about proper infant nutritional needs. Those who relied on breastmilk alone after six months, saw the greatest deficiency in protein-energy which is needed for cell creation.

According to Prado and Dewey (2014), studies of human autopsies and magnetic resonance imaging (MRI) showed infants with protein-energy deficiencies had fewer brain cells and lower cerebral cortical grey matter than normal infants. Also, autopsies of malnourished three to four-month-old infants had decreased dendritic spans. Because of the inhibited dendritic growth, infants of age 6 to 18 months with protein energy malnutrition showed lower motor performance and signifying delayed gross motor development (Kulkarni \& Metgund, 2014).

As previously mentioned, decreased dendritic spans leads to decreased synapses functioning and cognitive impairment. A 40-year longitudinal case-control study, by Galler et al., 2012, measured cognitive, health, and mental health outcomes of children, adolescents, and adults that suffered protein-energy malnutrition during their first year of life. Although the study population was nutritionally rehabilitated, lower IQ and academic achievement, conduct problems, depressive symptoms, and lower socioeconomic status resulted.

The participants in the Galler et al. (2012) study were from Barbados, born between 1967 and 1972, and were born with an Apgar score of eight or higher. They were then entered into the study after being admitted during their first year of life due to protein-energy malnutrition. To continue on in the study, the children were mandated to be in a government-supported health and nutrition program until age 12. Regular home visits and subsidized food were provided. Assessments of standard of living were taken during childhood and adulthood. Childhood standard of living was gauged by the Ecology Questionnaire which factored in 50 questions about education, employment of parents, and household items and utilities available for living. Adult standard of living was gauged by the Hollingshed Scales and the Occupation and Education Scales. Both assessed occupation and education. The Wechsler Intelligence Scale for Children was used to assess, at three different times, IQ of the children and adolescents. Longitudinal multiple regression analysis measured the outcomes of the scales and questionnaires throughout the 40 years.

The results of the Galler et al. (2012) study showed cognitive impairment throughout the 40 years as a result of the protein malnourishment before age two. Despite factoring in the socioeconomic and educational status of their parents, participants in the study still showed more of a decline in mental, emotional, and socioeconomic status than did their control group counterparts. Also, the study showed that measures taken to insure the future nourishment of the children did not reverse the damage done in the first year of life. This critical period for brain development needs protein energy to create brain cells. Without proper cell creation, brain development is inhibited. Protein, as well as other macronutrients, have many essential roles during the developmental stage that compound the lasting effects of the deficiencies.

\section{Fatty Acid Deficiency during Gestation and Infancy}

While protein energy is needed for the creation of cells, fatty acids are also of great importance during the first 1000 days. Both proteins and phospholipids, or fatty acids, are needed for the creation of myelin. Myelin is the protective fatty layer around neuron that allow a protected area for electrical impulses. These impulses are brain communications (Peters et al., 2014). According to Prado and Dewey (2014), the creation on neurons in the first 
years of life requires a great amount of membrane phospholipids. Docosahexaenoic acid (DHA), a fatty acid, in the membranes of synaptic sites help in not only the production of neurons but the maturation of synapses and subsequent neurotransmissions. Fatty acids accumulate rapidly in the third trimester of pregnancy. Preterm birth inhibits this accumulation and creates a deficiency in infants.

A study by Brew et al. (2015) found a positive relationship between lower plasma fatty acid levels and lower scores on standardized school tests through the first 14 years of life. The same study, however, did not find that supplementation of the fatty acids was effective early in life to reduce the negative learning impacts. An association was found between plasma omega-3 levels at age 8 and academic performance from 8-14 years old.

The Brew et al. (2015) researchers assessed the data from the Childhood Asthma Prevention Study and the supplementation of fatty acids through fish old capsules. Daily fish old supplements were added to infant's formula the cooking oils and spreads when they became older. The goal was that the infants and children received the same about of EPA and 1.5 times the DHA found in breast milk. The supplementation occurred for five years. Blood plasma phospholipid levels were assessed at ages 18 months, 3 years, 5 years, and 8 years.

The data in the Brew et al. (2015) study was compared to the scores from standardized tests the participants took during from ages 8-15. While the supplementation of DHA, EPA, and omega-3 fatty acids did not reverse the cognitive damage present from an early nutrient deficiencies, a positive relationship between the early deficiencies and subsequent low cognitive performance was found.

\section{Possible Interventions for Nutrient Deficiencies in the First Two Years of Life}

According to Prado and Dewey (2014), recovery of brain alterations from gestational and infant nutrient deficiencies is possible if the nutrients become available while the growth process is still happening. Also, adding sensory, linguistic, and social stimuli may help with the growth and recovery of brain functioning.

Black et al. (2011) suggested an intervention with iron supplementation, developmental interventions, and increased mother-child interactions. They cited a study in which the iron levels improved with the supplementation interventions but the socioemotional development continued to worsen without the increased mother-child interactions. This may be due to the iron-related depression in the prenatal mothers resulting in lower dopamine receptor functioning in the children.

Prado and Dewey (2014) suggested supplementation of DHA in the third trimester and through the first year could improve myelination, specifically of the brainstem auditory pathway. This is further supported by a Unay et al. (2004) study in which rapid brainstem auditory responses increased with infants that were on a DHA supplemented for formula through 16 weeks after birth. The infants without the DHA supplementation did not show the same auditory-evoked potentials. Furthermore, a positive effect on vocabulary and IQ have even been found at ages 5-6 years for DHA-supplemented, formula-fed infants (Prado \& Dewey, 2014).

Despite the positive effects found with infant formula DHA supplementation, Brew et al. (2015) found no relationship between the supplementation and an increase in standardized tests scores in subsequent years. A Dalton et al. (2009) study suggests supplementation of omega-3 from a fish flour could help improve cognitive function. In a randomized control study of school-aged children in South Africa, students who ate the fish flour, direct from marine sources, each school day for six months showed considerable cognitive improvements, both memory and learning processes, compared to those children who did not eat the fish flour. The results of this study suggests natural sources of fatty acids is more effective than supplementation capsules or powders.

Prado and Dewey (2014) suggested mental health issues that arise from nutritional deficiencies during infancy may not be entirely because of insufficient brain development. Caregivers may treat children different because of their smaller stature and not use age-appropriate interactions which will lead to slowed brain development. Also, malnourished infants and toddlers tend to be more fussy, irritable, and withdrawn which may lead the caregiver to disengage. The disengagement of the caregiver has been shown to contribute to poor brain development.

\section{Conclusion:-}

Despite world health campaigns, moderate to severe malnutrition in the first year of life has been linked to attention problems, lower social status, and standard of living, even 37-43 years later (Prado and Dewey, 2014). Furthermore, 
protein-energy deficiencies have been found to produce lower IQ and academic achievement, conduct problems, depressive symptoms, and lower socioeconomic status (Galler et al., 2012). And while interventions have been suggested that involve baby formula supplemented with iron and fatty acids and balanced diets high in protein after the discovery of the deficiencies, studies show there may not be the chance to reverse the damage done during the crucial brain developmental stage. There is evidence, though, to support the integration of these supplementations with positive caregiver interactions to help narrow the gap of developmental delays caused by iron, protein, and fatty acid deficiencies during gestation to two years of age.

\section{References:-}

1. Black, M. M., Quigg, A. M., Hurley, K. M., \& Pepper, M. R. (2011). Iron deficiency and iron-deficiency anemia in the first two years of life: strategies to prevent loss of developmental potential. Nutrition reviews, 69(suppl 1), S64-S70.

2. Black, R. E., Alderman, H., Bhutta, Z. A., Gillespie, S., Haddad, L., Horton, S., ... \& Maternal and Child Nutrition Study Group. (2013). Maternal and child nutrition: building momentum for impact. The Lancet, 382(9890), 372-375.

3. Brew, B. K., Toelle, B. G., Webb, K. L., Almqvist, C., \& Marks, G. B. (2015). Omega-3 supplementation during the first 5 years of life and later academic performance: a randomised controlled trial. European Journal of Clinical Nutrition, 69(4), 419-424.

4. Dalton, A., Wolmarans, P., Witthuln, R.C., van Stuijvenberg, M.E., Swanevelder, S.A., \& Smuts, C.M. (2009). A randomized control trial in school children showed improvement in cognitive function after consuming a bread spread containing fish flour from a marine source. Prostagland Leukot Essent Fatty Acids, 80, 143-149.

5. Galler, J. R., Bryce, C., Waber, D. P., Zichlin, M. L., Fitzmaurice, G. M., \& Eaglesfield, D. (2012). Socioeconomic outcomes in adults malnourished in the first year of life: a 40-year study. Pediatrics, 130(1), e1e7.

6. Jacka, F. N., Ystrom, E., Brantsaeter, A. L., Karevold, E., Roth, C., Haugen, M., ... \& Berk, M. (2013). Maternal and early postnatal nutrition and mental health of offspring by age 5 years: a prospective cohort study. Journal of the American Academy of Child \& Adolescent Psychiatry, 52(10), 1038-1047.

7. Lackner, C. L., Bowman, L. C., \& Sabbagh, M. A. (2010). Dopaminergic functioning and

8. preschoolers' theory of mind. Neuropsychologia, 48(6), 1767-1774.

9. Murashima, M., Hoerr, S. L., Hughes, S. O., \& Kaplowitz, S. A. (2012). Feeding behaviors of low-income mothers: directive control relates to a lower BMI in children, and a nondirective control relates to a healthier diet in preschoolers. The American Journal of Clinical Nutrition, 95(5), 1031-1037.

10. Knickmeyer, R. C., Gouttard, S., Kang, C., Evans, D., Wilber, K., Smith, J. K., ... \& Gilmore, J. H. (2008). A structural MRI study of human brain development from birth to 2 years. The Journal of Neuroscience, 28(47), 12176-12182.

11. Kulkarni, A., \& Metgud, D. (2014). Assessment of Gross Motor Development in Infants of Age 6 to 18 Months with Protein Energy Malnutrition Using Alberta Infant Motor Scale: A Cross Sectional Study. International Journal of Physiotherapy Res, 2(4), 616-20.

12. Peters, B. D., Voineskos, A. N., Szeszko, P. R., Lett, T. A., DeRosse, P., Guha, S., ... \& Malhotra, A. K. (2014). Brain white matter development is associated with a human-specific haplotype increasing the synthesis of long chain Fatty acids. The Journal of Neuroscience, 34(18), 6367-6376.

13. Prado, E. L., \& Dewey, K. G. (2014). Nutrition and brain development in early life. Nutrition reviews, 72(4), 267-284. 